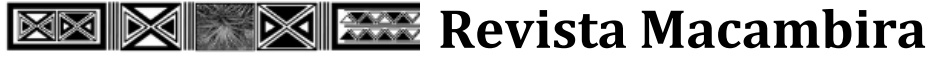

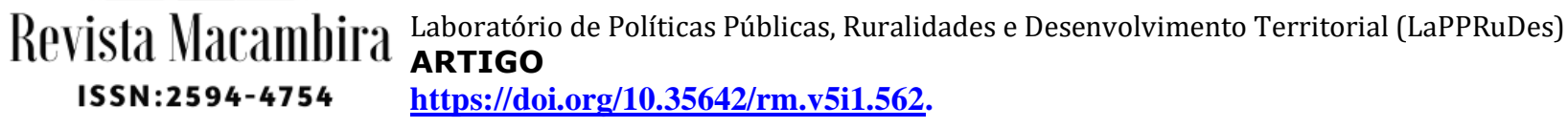

\section{Estado da arte no monitoramento acústico de Cicadidae em lavouras de café}

João Paulo Lemos Escola ${ }^{1 *}$, Rodrigo Capobianco Guido ${ }^{2}$, Ivan Nunes da Silva ${ }^{3}$, Douglas Henrique Bottura Maccagnan ${ }^{4}$, Alexandre Moraes Cardoso ${ }^{5}$, Uender Barbosa de Souza ${ }^{6}$

${ }^{1}$ Professor do Instituto Federal de São Paulo (IFSP) Campus Barretos, Mestre em Física Aplicada Computacional pelo Instituto de Física de São Carlos da Universidade de São Paulo (USP), Doutorando em Engenharia Elétrica pela Escola de Engenharia de São Carlos - USP. https://orcid.org/0000-0003-2787-6849

${ }^{2}$ Professor da Universidade Estadual Paulista (UNESP) Campus São José do Rio Preto, Mestre em Engenharia Elétrica pela Faculdade de Engenharia Elétrica e de Computação da Universidade Estadual de Campinas (UNICAMP), Doutor em Física Aplicada Computacional pelo Instituto de Física de São Carlos da USP, Livre Docente pela Escola de Engenharia de São Carlos da USP.

https://orcid.org/0000-0002-0924-8024

${ }^{3}$ Professor da USP Campus São Carlos, Mestre e Doutor em Engenharia Elétrica pela UNICAMP. https://orcid.org/0000-0002-1296-5454

${ }^{4}$ Professor da Universidade Estadual de Goiás (UEG) Unidade de Iporá, Mestre em Agronomia pela UNESP Campus Jaboticabal, Doutor em Entomologia pela USP Campus Ribeirão Preto. https://orcid.org/0000-0001-8557-1766

5Professor do IFSP Campus Barretos, Mestre em Entomologia Agrícola pela UNESP Campus Jaboticabal, Doutor em Entomologia USP Campus Ribeirão Preto.

https://orcid.org/0000-0002-4826-1141

6Professor do Instituto Federal de Goiás, Campus Goiânia, Mestre em Matemática pela Universidade Federal de Goiás (UFG), Doutorando em Engenharia Elétrica pela Universidade Federal de Goiás (UFG).

https://orcid.org/0000-0002-9549-1361

*Autor correspondente:

jpescola@ifsp.edu.br

\section{Resumo:}

As cigarras são uma praga-chave das lavouras de café com a característica marcante de emissão de sons. É de interesse do produtor o desenvolvimento de ferramentas para monitoramento das lavouras, buscando economia de recursos. Este trabalho realiza um levantamento bibliográfico dos trabalhos focados no monitoramento acústico de Cicadidae procurando soluções disponíveis para auxílio do produtor. Os resultados mostraram que a maioria dos trabalhos encontrados (52,3\%) utiliza softwares em laboratório e apenas 4,7\% utiliza algum software para monitoramento implantado em campo. Conclui-se, a partir desse dado, que essa pode ser uma importante lacuna a ser preenchida em trabalhos futuros, por meio do processamento digital de sinais de áudio em um possível dispositivo implantado em lavoura.

Palavras-chave: Bioacústica, Processamento Digital de Sinais de Áudio, Monitoramento.
REVISTA MACAMBIRA

Instituto Federal de Educação, Ciência e Tecnologia Baiano, campus Serrinha. Estrada Vicinal de Aparecida, s/n, Bairro Aparecida, Serrinha (Ba), CEP: 48700-000, sala 01, prédio acadêmico. 


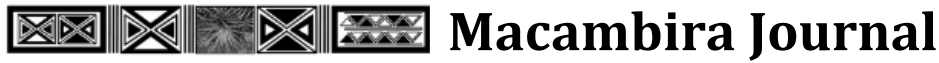

\author{
Revista Macamhilia laboratory of Public Policies, Ruralities and Territorial Development (LaPPRuDes) \\ ISSN:2594-4754 \\ https://doi.org/10.35642/rm.v5i1.562.
}

\section{State of the art in acoustic monitoring of Cicadidae in coffee crops}

\author{
João Paulo Lemos Escola ${ }^{1 *}$, Rodrigo Capobianco Guido ${ }^{2}$, Ivan Nunes da Silva ${ }^{3}$, Douglas Henrique
} Bottura Maccagnan ${ }^{4}$, Alexandre Moraes Cardoso ${ }^{5}$, Uender Barbosa de Souza ${ }^{6}$

${ }^{1}$ Professor at the Federal Institute of São Paulo (IFSP) Campus Barretos, Master in Computational Applied Physics from the Institute of Physics of São Carlos University of São Paulo (USP), PhD student in Electrical Engineering from the School of Engineering of São Carlos (USP).

https://orcid.org/0000-0003-2787-6849

${ }^{2}$ Professor at the Paulista State University (UNESP) Campus São José do Rio Preto, Master in Electrical Engineering from the Faculty of Electrical and Computer Engineering of the State University of Campinas (UNICAMP), $\mathrm{PhD}$ in Computational Applied Physics from the Institute of Physics of São Carlos of USP, Free Professor at the São Carlos School of Engineering, University of São Paulo of USP. https://orcid.org/0000-0002-0924-8024

${ }^{3}$ Professor at the University of São Paulo (USP) Campus São Carlos, Master and PhD in Electrical Engineering from the State University of Campinas (UNICAMP). https://orcid.org/00000002-1296-5454

4Professor at the State University of Goiás (UEG) Unit of Iporá, Master in Agronomy from the UNESP Jaboticabal Campus, PhD in Entomology from the USP Ribeirão Preto Campus.

https://orcid.org/0000-0001-8557-1766

5Professor at the Federal Institute of São Paulo (IFSP) Campus Barretos, Master in Agricultural Entomology from the Paulista State University (UNESP) Jaboticabal Campus, PhD in Entomology from the USP, Ribeirão Preto Campus.

https://orcid.org/0000-0002-4826-1141

6 Professor at the Federal Institute of Goiás, Campus Goiânia, Master in Mathematics from the Federal University of Goiás (UFG), PhD student in Electrical Engineering from the Federal University of Goiás (UFG).

https://orcid.org/0000-0002-9549-1361

${ }^{*}$ Corresponding author:

jpescola@ifsp.edu.br

\section{Abstract:}

Cicadas are a key pest of coffee plantations with a marked sound emission characteristic. It is of interest to the producer to develop tools to monitor crops in order to save resources. This research performs a bibliographic survey of works focused on the acoustic monitoring of Cicadidae, in order to look for available solutions to help producers. The results showed that the majority of the works founded (52.3\%) are related to the use of softwares in laboratory, and only $4.7 \%$ use some monitoring software implemented in the field. Therefore, that must be an important gap to be filled in future works, applying digital audio signal processing in a possible device implanted in a field.

Keywords: Bioacoustics, Digital Audio Signal Processing, Monitoring.
MACAMBIRA JOURNAL

Federal Institute of Education, Science and Technology Baiano, campus Serrinha. Estrada Vicinal de Aparecida, s/n, Bairro Aparecida, Serrinha, Bahia, Brasil, CEP: 48700-000, sala 01, prédio acadêmico. 


\section{Introdução}

Com o aumento da população humana, também é crescente a necessidade de produção de alimentos e de outros produtos advindos de campos agrícolas. Visando suprir tal necessidade, é comum encontrar monocultivos em áreas extensas, fato que pode contribuir com a diminuição da biodiversidade e favorecer a ocorrência de espécies de insetos-praga. Geralmente o manejo é feito com o uso de defensivos químicos, aplicados muitas vezes até em áreas onde a ocorrência das espécies de insetos-praga ainda sequer atingiu a densidade populacional capaz de promover algum prejuízo, o que onera o custo de produção e pode causar impactos ao ambiente e à saúde humana. $\mathrm{O}$ desenvolvimento de hardwares e softwares específicos para a detecção da praga no campo pode fornecer subsídios para o desenvolvimento de uma forma de produção de menor impacto. Nesse sentido, a captação, registro e análise de sinais acústicos emitidos por insetos pode ser uma alternativa para otimizar a produção de certos cultivos (MANKIN et al., 2011).

Um bom exemplo de inseto capaz de emitir sinais acústicos é a cigarra (Hemiptera: Cicadidae). Em condições brasileiras, a cultura do cafeeiro pode ser atacada por diversos artrópodes e, entre eles, Quesada gigas é considerada praga-chave em todo o estado de Minas Gerais e na região nordeste do estado de São Paulo (MARTINELLI; ZUCCHI, 1997). O fato de que há relatos de ocorrência de cigarras em cafezais paulistas desde o período entre 1900 e 1904 (D’UTRA, 1908) interfere na forma de condução da lavoura, o que praticamente obriga os cafeicultores a adotarem práticas de aprimoramento do sistema produtivo, como o aumento do espaçamento entre plantas para permitir a mecanização da lavoura, visando o uso de defensivos para manejo das cigarras e de outras pragas e doenças que afetam a produtividade da cultura.

As cigarras atacam as lavouras a procura de seiva, seu principal alimento. Os impactos sobre a planta acontecem na fase ninfal da cigarra, quando a praga suga seiva da raiz da planta hospedeira (SOUZA; REIS; SILVA, 2007). A espécie Q. gigas, a de maior tamanho no país, pode ultrapassar os 70 $\mathrm{mm}$ de comprimento (incluindo as asas) e $20 \mathrm{~mm}$ de largura no caso dos machos, já as fêmeas são um pouco menores, razão pela qual sua associação com o cafeeiro causa tanto impacto.

Nas regiões onde atacam a cultura de café, os machos costumam emitir som de outubro a dezembro. $\mathrm{Na}$ década de 1970, como não havia método eficiente de controle das cigarras, muitos cafeicultores não tinham outra opção senão erradicar as lavouras infestadas, o que culminava no abandono da cultura por diversos produtores (SOUZA; REIS; SILVA, 2007).

Nas últimas décadas, o controle recomendado é por meio de defensivos químicos sistêmicos (MARTINELLI et al., 1998). Mais recentemente, mostrando-se importante alternativa tecnológica, o uso de uma armadilha sonora que atrai a $Q$. gigas para um sistema fechado de pulverização tem sido uma opção (MACCAGNAN, 2008). 
Este artigo tem como objetivo realizar um levantamento bibliográfico, abordando as técnicas de monitoramento de Cicadidae em lavouras e florestas, com foco no uso de softwares e dispositivos eletrônicos, especialmente os que empregam processamento digital de sinais de áudio, permitindo a sua análise através do computador, e os que empregam redes neurais artificiais, que permitem o reconhecimento automático dos indivíduos na lavoura.

O trabalho está dividido da seguinte forma: a seção 2 apresenta uma revisão dos conceitos mais importantes; a seção 3 mostra um levantamento do estado da arte, a seção 4 expõe os resultados obtidos. Por fim, a seção 5 traz as conclusões e propostas para trabalhos futuros.

\section{Revisão dos Conceitos}

A característica mais marcante das cigarras é a emissão de sinais acústicos. Apenas o macho tem órgão específico para a geração desses sinais. O trabalho de Pringle (1954) foi o primeiro a fornecer detalhadamente uma análise anatômica e fisiológica do processo de geração de sinais acústicos por cigarras. De acordo com o autor, a onda sonora é gerada pela deformação do tímbalo, uma placa com propriedade elástica localizada dorso-lateralmente em cada uma das laterais do abdome. No interior do abdome, há espaços preenchidos por ar que atuam sobre a onda sonora como uma caixa de ressonância. A morfologia do tímbalo, a frequência que ele é deformado, aspectos morfológicos e movimentos do abdome no momento da emissão do sinal propiciam as características do sinal emitido, que é único para cada espécie.

Segundo Sueur (2001), há exemplos de espécies de cigarras que emitem sinais com frequência pouco abaixo de $1 \mathrm{kHz}$ até espécies com frequências ultrassônicas próximas a $25 \mathrm{kHz}$. A alta intensidade com as quais os sinais são emitidos também é uma característica marcante das cigarras, sendo que, em algumas espécies, ela pode ser superior a 100dB (VILLET, 1987). O fato de o sinal emitido pela cigarra ser espécie-específico faz dele um importante carácter taxonômico para fins de identificação, levantamento de riqueza de espécie e estimativa de tamanho populacional (SUEUR, 2001).

Embora o sinal acústico possa ter outras funções, como de espantar predadores, sua principal função é a de atrair a fêmea para o acasalamento (CLARIDGE, 1985). Após a cópula, a fêmea deposita seus ovos no interior de ramos da parte aérea da planta (JUNIOR et al., 2012). A ninfa recém eclodida vai ao solo, onde enterra-se em busca da raiz da planta da qual sugará seiva. É no subsolo, associada à raiz da planta, que a ninfa permanecerá por algum tempo (em alguns casos por vários anos), até pouco antes de completar seu desenvolvimento, quando ela retorna à superfície e fixa-se a um substrato vertical onde passará pelo processo de metamorfose e se tornará adulta, deixando fixada ao substrato sua última exúvia (TRIPLEHORN; JOHNSON, 2011). 


\section{Estado da Arte}

Foram analisados diversos artigos que abordam assuntos relacionados ao monitoramento, controle, detecção das cigarras em seus mais diversos ambientes. A partir desses estudos, buscou-se, principalmente, relacionar os processos com o uso de tecnologias, como softwares e dispositivos tecnológicos, com duas finalidades: i) investigar se os agricultores empregam tais artifícios em suas rotinas de cultivo de espécies vegetais que têm incidência de cigarras como praga; e ii) fazer um levantamento do estado da arte em relação a busca de soluções para o problema.

O trabalho de Gonsalves e Faria (1989) analisa a eficiência de inseticidas granulados no controle de ninfas móveis em cafeeiros. O levantamento populacional, a exemplo de outros que serão citados posteriormente, foi feito por meio de observação direta e contagem não automatizada de insetos. Particularmente nesse caso, de forma aleatória, nos talhões, foram abertas trincheiras $(1,5 \mathrm{~m} \mathrm{X} \mathrm{1,5} \mathrm{m} \mathrm{X}$ $0,5 \mathrm{~m})$ no centro da cova e o solo retirado foi peneirado, assim os insetos foram coletados e contatos pelos pesquisadores.

De acordo com Reis e Souza (1991), é possível detectar a presença de cigarras na lavoura analisando o estado das plantas, cujos sintomas são: "clorose das folhas das extremidades dos ramos, queda precoce de folhas, com permanência de folhas no ápice” (MARTINELLI, 1998, p. 133), confirmando que a solução mais eficiente é o controle químico com inseticidas.

Já em Martinelli e Zucchi (1997), o monitoramento é feito por armadilha constituída de uma armação de ferro coberta com tela de náilon, sob a copa do café. Nesse caso, também foram realizadas coletas manuais.

No artigo de Fornazier (2000), foi analisada a eficiência de diversas formas de aplicação de inseticidas. Para tanto, foram feitas covas sob as plantas para realizar a contagem manual das ninfas e estimativa de sua população inicial. A avaliação dos resultados também foi realizada de forma manual, não só com a contagem do número de ninfas ainda vivas após o procedimento, mas também com a medida da produtividade de 50 das plantas submetidas ao processo. Nesse artigo, fica registrado que o incremento da produtividade ocorreu somente após o terceiro ano de aplicação de defensivos.

Em Souza, Reis e Silva (2007), o monitoramento da infestação de cigarras na cultura do café é feito manualmente e consiste em entrincheirar covas nas plantações à procura de ninfas nas raízes ou em verificar a presença de exúvias ao longo da base do caule. Esse processo é realizado com a escolha de 10 plantas aleatórias em um talhão, que são entrincheiradas de um único lado de fora para dentro. Terminado o processo, as ninfas capturadas devem ser contadas e o resultado multiplicado por 2, em razão de o processo ser realizado somente de um dos lados da planta. O autor recomenda que, caso o resultado seja de 35 ninfas ou mais, deve-se realizar o controle químico. 
Em Santos e Martinelli (2007), foi feito o registro de uma espécie de cigarras no município de Tapiratiba, estado de São Paulo. Nesse estudo, os exemplares foram coletados manualmente com rede entomológica, que consiste em um aro com rede, preso a um cabo de madeira ou de aço.

Em Soares et al. (2008), foi analisada a distribuição de ninfas de cigarras em plantações de paricá (Schizolobium amazonicum Huber ex Ducke) por meio de interpolação de unidades amostrais. A partir do mapa da localidade, foi utilizado um software para dividir a área, a fim de encontrar pontos para coletas de amostragens. A contagem das cigarras foi feita de forma não-automatizada, pela observação direta dos insetos, e os dados geraram mapas de dispersão dos indivíduos no solo com o objetivo de conhecer sua incidência dentro da região de estudo.

Lee (2008) apresenta uma pesquisa em que é feito o levantamento das espécies de cigarras na península Coreana. Os sons das cigarras são capturados e analisados por oscilogramas e sonogramas. Os dados são comparados, não com intenção de detecção, mas sim com a de diferenciação das espécies.

No trabalho publicado por Cole (2008), a captura de sons é feita com um microfone modelo C451E e com um gravador DAT. A maioria das gravações aconteceu a 1 metro ou mais de distância. As frequências foram analisadas por espectrogramas gerados a partir da Transformada Rápida de Fourier (FFT). Nesse caso, uma comparação é feita entre duas espécies, analisando os gráficos dos sons dos machos.

Em Chen e Shiao (2008), são apresentadas duas novas espécies de cigarras. A metodologia consistiu na captura dos sons emitidos em campo, realizada por um gravador digital Foster FR-2 e por um microfone Sennheiser MKH 70 P48. O microfone foi colocado a cerca de 5 metros dos insetos em geral. O espectro de frequências é analisado para diferenciar as espécies.

Outro trabalho importante é o de Sueur et al. (2008), que propõe uma solução econômica e não invasiva para o desenvolvimento de inventários RBA (Rapid Biodiversity Assessments), utilizando o som da comunicação das espécies para estimar a população em determinada localidade. Foram aplicados, como recursos, o índice de Shannon e a Transformada de Fourier de Tempo Curto (STFT). As gravações foram realizadas utilizando um microfone omni-direcional Sennheiser Me62/K6 conectado a um gravador digital Edirol R-09, com sinais de 30 segundos cada. O estudo foi aplicado em 45 espécies, sendo 15 de aves, 15 de anfíbios e 15 de insetos. Nos experimentos, foram criados sons, juntando coros de várias espécies, para verificar a eficácia do sistema em ambientes onde elas vivem em conjunto.

Em Seabra, Quartau e Bruford (2009), foi feita uma pesquisa sobre a variação de espécies de cigarras na região do Mar Mediterrâneo. São citadas questões importantes, como a similaridade morfológica e acústica entre as espécies Cicada barbara e Cicada orni. Entretanto, a pesquisa ficou restrita à variação genética e geográfica das espécies, não sendo aplicados estudos relacionados ao som. Os espécimes foram coletados manualmente ou encaminhados por instituições parceiras e estudados em laboratório. Os estudos estatísticos foram realizados por meio de softwares específicos para esse fim. 
Já Sanborn, Heath e Heath (2009) analisam os sons emitidos pela cigarra Beameria venosa. Essa espécie emite uma alta frequência, que se torna uma vantagem, pois seus predadores (pássaros e lagartos) não a ouvem, assim há uma baixa pressão predatória. Os sons foram captados em fitas, em um dispositivo Uher 4000. O microfone foi colocado o mais próximo possível para evitar interferências externas. As gravações foram realizadas em diferentes cidades dos Estados Unidos (EUA).

O método do entrincheiramento também é empregado em Lunz et al. (2010), utilizando implemento tratorizado para raspagem de solo. Com auxílio de hastes metálicas, são feitas verificações à procura de ninfas vivas. Posteriormente são aplicados inseticidas e é feita a comparação de populações entre amostragens antes e depois de sua aplicação.

Já no artigo Dardar, Belal e Basheer (2013), as cigarras adultas foram coletadas manualmente das macieiras (Malus domestica) ao pôr do sol e ao amanhecer, período de maior atividade biológica, o que torna a coleta mais fácil. Para tanto, foi utilizada uma armadilha com uma estrutura em formato de tenda com tela de pano. As ninfas foram coletadas por escavação de 10 a $15 \mathrm{~cm}$. Nesse trabalho também foram coletados os sons, posteriormente analisados pelo software Speech Analyzer. Os ciclos de frequência também foram analisados para registrar as características dos indivíduos frente ao ambiente de estudo.

No caso de Hill e Marshall (2013), a pesquisa versou sobre um gênero de cigarras comum nos Estados Unidos (EUA). Para capturar o som dos insetos, foram utilizados um gravador digital e um microfone. Um GPS (Global Positioning System) manual foi utilizado para precisar a posição geográfica. Os sons foram analisados em laboratório utilizando softwares, e a temperatura, aferida manualmente, foi catalogada.

A identificação de insetos pelo uso do som emitido foi efetivamente aplicada em Lampson et al. (2013) com o objetivo de diminuir tempo e custos com defensivos agrícolas. O processo foi realizado por meio de um acelerômetro modelo 4370, Bruel \& Kjaer, pois o sinal emitido pelo inseto estudado é o sinal vibratório propagado através do substrato. No estudo, foram aplicados os métodos de identificação GMM (Gaussian mixture model) e PNN (Probabilistic neural network). Os testes foram realizados em laboratório com cerca de 100 indivíduos, alguns criados em laboratório e outros previamente capturados. Os resultados obtidos foram de $92 \%$ de detecção com testes envolvendo pares de macho-fêmea e $86 \%$ com conjuntos de 30 indivíduos.

No trabalho de Aide et al. (2013), foi desenvolvido um sistema de coleta de dados, monitoramento e identificação baseado em gravações de áudio. O sistema é composto por softwares, além de uma estação de monitoramento remota alimentada por energia solar. Essa estação envia 1 min de gravação a cada 10 min de intervalo. As gravações de áudio são coletadas por um microfone com resposta em frequência de $20 \mathrm{~Hz}$ a $20 \mathrm{kHz}$ conectado a um dispositivo iPod. O processamento dos arquivos é feito por meio de uma STFT. A detecção é realizada por meio de um algoritmo que utiliza HMMs (Hidden Markeov Models). 
No artigo de Maccagnan et al. (2014), são apresentadas evidências da detecção de Cicadidae, por meio da constatação a olho nu da presença de exúvias presas ao tronco das árvores bem como galerias de ninfas junto ao sistema radicular. A catação manual foi realizada para os indivíduos adultos presentes no tronco das árvores.

Em Hou et al. (2015), o monitoramento é realizado pelo processo de escavação em três tipos de plantas Pinus tabuliformis Carr. (Pinaceae), Populus tomentosa Carr. (Salicaceae) e Pyrus xerophila Yu (Rosaceae). A distribuição das ninfas em solo é feita pela contagem manual a cada $10 \mathrm{~cm}$ de profundidade do solo. A coleta de exúvias foi realizada diariamente às 9h da manhã durante os anos 2013 e 2014.

Sato e Sato (2015) realizam o monitoramento de ninfas no Japão para verificar o impacto da mudança de ambiente. A coleta foi realizada manualmente e por uma rede com cabo de $4 \mathrm{~m}$ para coleta em lugares mais elevados. A contagem foi realizada em laboratório.

Por fim, no caso de Chiavacci e Bednarz (2015), a detecção das cigarras foi realizada por meio de armadilhas com câmeras, para analisar a densidade populacional de cigarras na região, além de estudar a relação entre a incidência da população de cigarras e escolha da localização dos ninhos pelos papagaios, que são seus predadores naturais. A contagem foi realizada manualmente a cada 3 dias, sendo tanto as cigarras adultas quanto as exúvias armazenadas separadamente. A posição de cada armadilha foi registrada manualmente pelos pesquisadores utilizando um dispositivo GPS.

\section{Resultados}

No Quadro 1, temos a relação dos artigos estudados e uma análise dos tipos de abordagem. A primeira coluna apresenta a referência do artigo, a segunda coluna informa se houve aplicação de técnicas manuais; a terceira coluna (SC) discrimina os trabalhos em que há aplicação de sofwares em campo; a última coluna (SL) identifica os artigos em que há uso de softwares em laboratório.

Verifica-se que todos os artigos estudados (100\%) implementam técnicas manuais para monitoramento de cigarras e necessitam, portanto, da interferência humana no processo ou do trabalho manual de lavradores.

De todos os 21 trabalhos analisados, apenas um (4,8\%) utiliza softwares em campo (coluna SC), ou seja, existe um sistema computacional empregado durante o processo de monitoramento na lavoura. Nos demais, os dados são enviados ao laboratório para, posteriormente, serem processados computacional ou manualmente.

Já 11 dos 21 trabalhos (52,4\%) utilizam softwares em laboratório. Nesses casos, os pesquisadores fazem o monitoramento na lavoura e encaminham os dados brutos para serem analisados em laboratório para depois obterem um resultado. 
Quadro 1 - Relação de artigos consultados/estudados com as respectivas abordagens.

\begin{tabular}{|lccc|}
\hline \multicolumn{1}{|c|}{ Referência do Artigo } & Técnicas Manuais & SC & SL \\
\hline GONSALVES; FARIA, 1989 & Sim & Não & Não \\
REIS; SOUZA, 1991 & Sim & Não & Não \\
MARTINELLI; ZUCCHI, 1997 & Sim & Não & Não \\
FORNAZIER, 2000 & Sim & Não & Não \\
SOUZA; REIS; SILVA, 2007 & Sim & Não & Não \\
SANTOS; MARTINELLI, 2007 & Sim & Não & Não \\
LEE, 2008 & Sim & Não & Sim \\
COLE, 2008 & Sim & Não & Sim \\
CHEN; SHIAO, 2008 & Sim & Não & Sim \\
SUEUR et al., 2008 & Sim & Não & Sim \\
SEABRA; QUARTAU; BRUFORD, 2009 & Sim & Não & Sim \\
SANBORN; HEATH; HEATH, 2009 & Sim & Não & Sim \\
LUNZ et al., 2010 & Sim & Não & Não \\
DARDAR; BELAL; BASHEER, 2013 & Sim & Não & Sim \\
HILL; MARSHALL, 2013 & Sim & Não & Sim \\
LAMPSON et al., 2013 & Sim & Não & Sim \\
AIDE et al., 2013 & Sim & Sim & Sim \\
MACCAGNAN et al., 2014 & Sim & Não & Não \\
HOU et al., 2015 & Sim & Não & Não \\
SATO; SATO, 2015 & Sim & Não & Não \\
CHIAVACCI; BEDNARZ, 2015 & Sim & Não & Sim \\
\hline
\end{tabular}

Fonte: Os autores (2021)

\section{Discussões}

Poucos são os artifícios tecnológicos empregados nas lavouras cafeeiras para monitoramento e controle das cigarras, sendo o controle químico o método mais utilizado. Os métodos atualmente empregados para mapeamento e monitoramento de populações de cigarras consistem basicamente na contagem não automatizada dos indivíduos através da observação direta.

Não foram encontrados trabalhos que integram o uso de tecnologia autônoma no levantamento, detecção ou monitoramento de espécies. O desenvolvimento dessas tecnologias pode ser útil em plantios de café, perante a ameaça da cigarra, praga-chave desse tipo de cultura.

O uso de mão de obra humana no processo de coleta e análise de dados voltados ao monitoramento de cigarras ainda é o principal artifício empregado pelos agricultores, embora seja possível economizar tempo e dinheiro nesse processo com as tecnologias aqui sugeridas.

Os trabalhos que utilizam tecnologias de processamento de áudio ou de redes neurais o fazem com foco em coletas de informações e estatísticas utilizadas em laboratório e não em soluções voltadas ao mercado de cultivo do produto. Ou seja, para o agricultor, não há soluções disponíveis que facilitem 
o processo de verificação de incidência de pragas para tomada de decisão de controle, visando o uso racional da melhor estratégia e do melhor momento de sua implementação.

Um dispositivo automatizado capaz de detectar cigarras na lavoura de café pode ser uma solução importante para o desenvolvimento e aprimoramento do manejo da cultura. Esse método possibilitaria o conhecimento das áreas da lavoura onde há incidência dos insetos e auxiliaria na tomada de decisão para atuação de controle posterior.

Nos trabalhos de Gonsalves e Faria (1989), Reis e Souza (1991), Fornazier (2000) e Lunz et al. (2010), tal dispositivo auxiliaria no processo de estimativa populacional de indivíduos em determinada área, visto que a identificação dos indivíduos através de redes neurais pode ser aplicada a fim de verificar o nível (baixo, médio ou alto) de indivíduos perante a análise acústica do ruído audível emitido na área.

A perspectiva da identificação das cigarras por espécie, possível por meio do referido dispositivo, poderá auxiliar ainda mais no processo de tomada de decisão, que pode resultar na troca do procedimento de interferência física na lavoura ou na substituição da estratégia de manejo a ser empregada.

Uma rica promessa do procedimento proposto está na possibilidade de execução do processo de análise de biodiversidade, de forma automatizada, dos mais diversos grupos animais vertebrados e invertebrados, o que é essencial não só para o monitoramento de pragas agrícolas (BARZMAN et al., 2015), mas também para desenvolvimento de estratégias conservacionistas (PRIMACK; RODRIGUES, 2006).

Um dos pontos apresentados neste trabalho foi a importância de um levantamento da incidência de espécimes dentro da área de cultivo, o que pode ser resolvido com a inclusão de um módulo GPS dentro do dispositivo aqui proposto. Isso possibilita a indicação da exata posição geográfica ao mesmo tempo que se detecta a presença do indivíduo de estudo, fatos que compõem a Agricultura de Precisão, pois permitem a implementação das medidas de manejo somente nos pontos de ocorrência da praga e não em área total, como é realizado atualmente.

Por não terem como característica a emissão de sons, as ninfas não fazem parte do objeto de estudo deste projeto, que preconiza o uso de tecnologias de processamento de sinais de áudio em seus procedimentos de identificação e detecção. Entretanto, procedimentos que utilizem sinais infravermelhos ou captura ultrassônica podem ser empregados para o processo envolvendo as ninfas sob o solo, além da possibilidade de captar sinais vibratórios emitidos pela ninfa, no tronco da planta.

Outra possibilidade do processamento digital automatizado de sinais de áudio é o desenvolvimento de um dispositivo que emita um ruído que cause repelência aos indivíduos do presente objeto de estudo. Afastar os machos pode diminuir a incidência de acasalamentos, reduzindo a incidência da espécie na região onde o dispositivo está instalado.

Essas estratégias sem o uso de agroquímicos mostram-se promissoras na busca da redução da poluição do solo e do meio ambiente como um todo. O trabalho mais próximo dessa solução de controle 
de pragas na lavoura é o de Maccagnan (2008), em que uma armadilha foi desenvolvida para captura dos indivíduos. Essa armadilha consiste em uma corneta que emite o som da cigarra, atraindo os machos e as fêmeas de cigarras da espécie Quesada gigas, que são exterminadas por um jato de inseticida ao se aproximarem do dispositivo. O procedimento também foi testado substituindo o inseticida por água (procedimento conhecido como "testemunha").

\section{Considerações Finais}

O presente trabalho apresentou um levantamento do estado da arte dos estudos envolvendo Cicadidae em lavouras de café e os esforços em busca de soluções para o monitoramento da região de incidência, a fim de buscar soluções para seu manejo.

Diante dos fatos, um dispositivo de monitoramento de lavouras de café por meio da captura do som mostra-se um recurso pertinente, visto que não foi possível encontrar esse tipo de solução durante o processo de levantamento bibliográfico apresentado.

Em trabalhos futuros, pretende-se implementar um sistema para captura e processamento do sinal emitido pelo macho de Cicadidae com o objetivo de efetuar sua detecção e identificação utilizando redes neurais artificiais.

\section{Referências}

AIDE, T. M. et al. Real-time bioacoustics monitoring and automated species identification. PeerJ, PeerJ Inc., v. 1, p. 1-19, 2013. Disponível em: https://doi.org/10.7717/peerj.103. Acesso em: 16 jun. 2021.

BARZMAN, M. et al. Eight principles of integrated pest management. Agronomy for sustainable development, Springer, v. 35, n. 4, p. 1199-1215, 2015. https://doi.org/10.1007/s13593-015-0327-9. Acesso em: 16 jun. 2021.

CHEN, C.-H.; SHIAO, S.-F. Two new species of the genus Euterpnosia matsumura (Hemiptera: Cicadidae) from Taiwan. The Pan-Pacific Entomologist, BioOne, v. 84, n. 2, p. 81-91, 2008. Link: https://doi.org/10.3956/2007-02.1

CHIAVACCI, S. J.; BEDNARZ, J. C.; T., M. The emergence densities of annual cicadas (hemiptera: Cicadidae) increase with sapling density and are greater near edges in a bottomland hardwood forest. Environmental Entomology, v. 44, p. 859-867, 2015. ISSN 1938-2936. Link: https://doi.org/10.1603/en13338

CLARIDGE, M. Acoustic signals in the homoptera: behavior, taxonomy, and evolution. Annual review of entomology, Palo Alto, v. 30, n. 1, p. 297-317, 1985. Link: https://doi.org/10.1146/annurev.en.30.010185.001501

COLE, J. A. A new cryptic species of cicada resembling Tibicen dorsatus revealed by calling song (Hemiptera: Auchenorrhyncha: Cicadidae). Annals of the Entomological Society of America, BioOne, v. 101, n. 5, p. 815-823, 2008. Link: https://doi.org/10.1093/aesa/101.5.815 
DARDAR, M. A.; BELAL, H. M.; BASHEER, A. M. The occurrence of the cicada Cicadatra Persica on apple trees, malus domestica, in Erneh, Syria. Journal of Insect Science, v. 13, n. 1, p. 1-5, 2013. Disponível em: http://dx.doi.org/10.1673/031.013.4201. Acesso em: 16 jun. 2021.

D'UTRA, G. Cigarras nos cafezais. Boletim da Agricultura, São Paulo, v. 9, n. 5, p. 350-365, 1908.

FORNAZIER, M. J.; ROCHA, A. C. D. Controle da cigarra do cafeeiro em regiões declivosas no estado do Espírito Santo. In: Simpósio de Pesquisa dos Cafés do Brasil, 1., 2000, Poços de Caldas, MG. Resumos expandidos. Brasília, D.F.: Embrapa Café; Belo Horizonte: Minasplan, 2000. 2v. p. 1167-1170. Disponível em: http://www.sbicafe.ufv.br/handle/123456789/606. Acesso em: 16 jun. 2021.

GONSALVES, W.; FARIA, A. M. Inseticidas sistêmicos granulados no controle das ninfas móveis das cigarras e seus efeitos na produtividade de cafeeiros. Bragantia, Scielo, v. 48, p. 95-108, 1989. ISSN 0006-8705. Disponível em: http://dx.doi.org/10.1590/S0006-87051989000100009. Acesso em: 16 jun. 2021.

HILL, K. B.; MARSHALL, D. C. The song, morphology, habitat, and distribution of the elusive North American cicada Okanagana Viridis (Auchenorrhyncha: Cicadidae). Annals of the Entomological Society of America, BioOne, v. 106, n. 5, p. 598-603, 2013. Link: https://doi.org/10.1603/an13072.

HOU, Z. et al. Ecology of Meimuna Mongolica (hemiptera: Cicadidae) nymphs: Instars, morphological variation, vertical distribution and population density, host-plant selection, and emergence phenology. Journal of Insect Science, v. 15, n. 1, p. 1-6, 2015. Disponível em: http://dx.doi.org/10.1093/jisesa/iev031. Acesso em: 16 jun. 2021.

JUNIOR, S. T. D. et al. Oviposition of quesada gigas (hemiptera: Cicadidae) in coffee plants. Revista Colombiana de Entomología, Sociedad Colombiana de Entomología, v. 38, n. 1, p. 1-5, 2012. Link: http://www.scielo.org.co/pdf/rcen/v38n1/v38n1a01.pdf

LAMPSON, B. et al. Automatic detection and identification of brown stink bug, Euschistus Servus, and southern green stink bug, Nezara Viridula,(Heteroptera: Pentatomidae) using intraspecific substrateborne vibrational signals. Computers and electronics in agriculture, Elsevier, v. 91, p. 154-159, 2013. Link: https://doi.org/10.1016/j.compag.2012.12.010

LEE, Y. J. Revised synonymic list of Cicadidae (Insecta: Hemiptera) from the Korean peninsula, with the description of a new species and some taxonomic remarks. Proceedings of the Biological Society of Washington, BioOne, v. 121, n. 4, p. 445-467, 2008. Link: https://doi.org/10.2988/08-09.1

LUNZ, A. M. et al. Ocorrência de Pantophthalmus kerteszianus e P. chuni (Diptera: Pantophthalmidae) em paricá, no Estado do Pará. Pesquisa Florestal Brasileira, v. 30, n. 61, p. 71-71, 2010. Link: https://doi.org/10.4336/2010.pfb.30.61.71

MACCAGNAN, D. H. B. Cigarra (Hemiptera: Cicadidae): emergência, comportamento acústico e desenvolvimento de armadilha sonora. 2008. Tese (Doutorado em Entomologia) - Faculdade de Filosofia, Ciências e Letras, Universidade de São Paulo, São Paulo, 2008. Link: https://www.ffclrp.usp.br/imagens defesas/02 $05 \quad 2013 \quad 16 \quad 12 \quad 59 \quad$ 45.pdf

MACCAGNAN, D. H. B. et al. Primeiro registro de cigarra em reflorestamentos com paricá no Estado de Mato Grosso, Brasil. Revista de Ciências Agrárias Amazonian Journal of Agricultural and Environmental Sciences, v. 57, n. 4, p. 451-454, 2014. Link: https://doi.org/10.4322/rca.1387

MANKIN, R. et al. Perspective and promise: a century of insect acoustic detection and monitoring. American Entomologist, The Oxford University Press, v. 57, n. 1, p. 30-44, 2011. Link: https://doi.org/10.1093/ae/57.1.30

MARTINELLI, N. M. et al. Modo de aplicação e eficiência de inseticidas granulados sistêmicos para o controle de cigarras (Hemiptera: Cicadidae) do cafeeiro. Anais da Sociedade Entomológica do Brasil, v. 27, n. 1, p. 133-140, 1998. Link: https://doi.org/10.1590/s0301-80591998000100017 
MARTINELLI, N. M.; ZUCCHI, R. A. Cigarras (Hemiptera: Cicadidae: Tibicinidae) associadas ao cafeeiro: distribuição, hospedeiros e chave para as espécies. Anais da Sociedade Entomológica do Brasil, v. 26, n. 1, p. 133-143, 1997. Link: https://doi.org/10.1590/s0301-80591997000100018

PRIMACK, R. B.; RODRIGUES, E. Biologia da conservação. Ed. Planta, Londrina. 327p.

PRINGLE, J. A physiological analysis of cicada song. Journal of Experimental Biology, The Company of Biologists Ltd, v. 31, n. 4, p. 525-560, 1954. Link: https://doi.org/10.1242/jeb.31.4.525

REIS, P. R.; SOUZA, J. C. Cigarras-do-cafeeiro, dano e controle. Circular. EPAMIG / CRSM, Lavras, 1991.

SANBORN, A. F.; HEATH, J. E.; HEATH, M. S. Long-range sound distribution and the calling song of the cicada Beameria venosa (uhler) (hemiptera: Cicadidae). The Southwestern Naturalist, BioOne, v. 54, n. 1, p. 24-30, 2009. Link: https://doi.org/10.1894/jc-23.1

SANTOS, R. S.; MARTINELLI, N.M. Ocorrência de Fidicinoides pauliensis Boulard \& Martinelli, 1996 (Hemiptera: Cicadidae) em cafeeiro em Tapiratiba, SP. Revista de Agricultura, Piracicaba, v. 82, p. 311314, 2007. Link: https://doi.org/10.37856/bja.v82i3.1465

SATO, Y.; SATO, S. Spring temperature predicts the long-term molting phenology of two cicadas, Cryptotympana facialis and Graptopsaltria nigrofuscata (hemiptera: Cicadidae). Annals of the Entomological Society of America, v. 108, n. 4, p. 494-500, 2015. Disponível em: http://dx.doi.org/10.1093/aesa/sav036. Acesso em: 16 jun. 2021.

SEABRA, S. G.; QUARTAU, J. A.; BRUFORD, M. W. Spatio-temporal genetic variation in sympatric and allopatric mediterranean cicada species (Hemiptera, Cicadidae). Biological Journal of the Linnean Society, Wiley Online Library, v. 96, n. 2, p. 249-265, 2009. Link: https://doi.org/10.1111/j.10958312.2008.01116.x

SOARES, V. C. et al. Análise espacial da distribuição de cigarras (Quesada gigas Oliver) em povoamentos de paricá (Schizolobium amazonicum Huber ex Ducke) na região de Dom Eliseu, PA. Revista Árvore, v. 32, n. 2, p. 251-258, 2008. Link: https://doi.org/10.1590/s0100-67622008000200008

SOUZA, J.; REIS, P. R.; SILVA, R. A. Cigarras-do-cafeeiro em Minas Gerais: histórico, reconhecimento, biologia, prejuízos e controle. 2. ed. Belo Horizonte, MG: EPAMIG, 2007. ISSN 0101-062X.

SUEUR, J. Audiospectrographical analysis of cicada sound production: a catalogue (Hemiptera, Cicadidae). Deutsche Entomologische Zeitschrift, Wiley Online Library, v. 48, n. 1, p. 33-51, 2001.

SUEUR, J. et al. Rapid acoustic survey for biodiversity appraisal. PloS one, Public Library of Science, v. 3, n. 12, p. 1-9, 2008. Link: https://doi.org/10.1371/journal.pone.0004065

TRIPLEHORN, C. A.; JOHNSON, N. F. Estudo dos insetos. São Paulo: Cengage Learning, 2011.

VILLET, M. Sound pressure levels of some African cicadas (Homoptera: Cicadoidea). Journal of the Entomological Society of Southern Africa, Entomological Society of South Africa (ESSA), v. 50, n. 2, p. 269-273, 1987. 


\begin{tabular}{|c|c|}
\hline Informações do Artigo & Article Information \\
\hline $\begin{array}{l}\text { Recebido em: } 23 / 03 / 2021 \\
\text { Aceito em: 16/06/2021 } \\
\text { Publicado em: 18/06/2021 }\end{array}$ & $\begin{array}{l}\text { Received on: } 23 / 03 / 2021 \\
\text { Accepted in: } 16 / 06 / 2021 \\
\text { Published on: } 18 / 06 / 2021\end{array}$ \\
\hline $\begin{array}{l}\text { Conflitos de Interesse: Os autores declaram não } \\
\text { haver quaisquer conflitos de interesse referente a } \\
\text { este artigo. }\end{array}$ & Conflict of Interest: No reported. \\
\hline Como citar este artigo & How to cite this article \\
\hline $\begin{array}{l}\text { Escola, J. P. L. et al., (2021). Estado da arte no } \\
\text { monitoramento acústico de Cicadidae em lavouras } \\
\text { de café. Revista Macambira, 5(1), e051007. } \\
\text { https://doi.org/10.35642/rm.v5i1.562. }\end{array}$ & $\begin{array}{l}\text { Escola, J. P. L. et al., (2021). State of the art in } \\
\text { acoustic monitoring of Cicadidae in coffee crops. } \\
\text { Revista Macambira, 5(1), e051007. } \\
\text { https://doi.org/10.35642/rm.v5i1.562. }\end{array}$ \\
\hline (c) & (c) \\
\hline $\begin{array}{l}\text { Este trabalho está licenciado sob uma Licença } \\
\text { Internacional Creative Commons Attribution- } \\
\text { NonCommercial-ShareAlike } 4.0 \text { International . }\end{array}$ & $\begin{array}{l}\text { This work is licensed under a Creative Commons } \\
\text { Attribution-NonCommercial-ShareAlike } \\
\text { International License. }\end{array}$ \\
\hline
\end{tabular}

